\title{
Managing an External Depot in a Production Routing Problem
}

\author{
Bi Kouaï Bertin Kayé ${ }^{1}$, Moustapha Diaby ${ }^{2}$, Tchimou N'Takpé ${ }^{3}$, Souleymane Oumtanaga ${ }^{4}$ \\ Institut National Polytechnique Félix Houphouët-Boigny, Yamoussoukro, Côte d'Ivoire ${ }^{1,4}$ \\ Ecole Supérieur Africaine des TIC, Abidjan, Côte d'Ivoire ${ }^{2}$ \\ Université Nangui Abrogoua, Abidjan, Côte d'Ivoire ${ }^{3}$ \\ Laboratoire de Recherche en Informatique et Télécommunication, Abidjan, Côte d'Ivoire ${ }^{1,2,3,4}$
}

\begin{abstract}
This paper addresses a production and distribution problem in a supply chain. The supply chain consists of a plant with no storage capacity that produces only one type of product. The manufactured products are then transported to a depot for storage. Customers demand is met by a homogeneous fleet of vehicles that begins and ends their trips at the depot. The objective of the study is to minimize the overall cost of production, inventory and transport throughout the supply chain. A Branch-and-Cut and a hybrid Two Phases Decomposition Heuristic using a Mixed Integer Programming and a Genetic Algorithm have been developed to solve the problem.
\end{abstract}

Keywords-Production; inventory; distribution; transport; branch-and-cut; decomposition heuristic; MIP; genetic algorithm

\section{INTRODUCTION}

In a supply chain, several activities are involved in meeting consumer needs. Producing, storing and distributing products are the main activities of the actors or companies that make up a supply chain. The problem of production or Lot Sizing (LSP) consists in determining the production schedule, the quantities to be produced when there is production and the quantities stored on the planning horizon. Planning production therefore means finding the best compromise between the production schedule and the quantities stored on the planning horizon. For a better visibility on production planning, readers are invited to see [1] . Another very important problem in the area of supply chain planning is the Vehicle Routing Problem (VRP). This is a NP-Hard problem as a particular case of the Travelling Salesman Problems (TSP) which is itself a NPHard problem [2]. It does not consider inventory and production decisions. It is mainly interested in the organization of the routes of each vehicle, knowing that the schedule for each customer's visit is known in advance. It therefore makes it possible to answer questions such as "Who to visit? "and "In which order should the visits be made? The objective of a VRP is to minimize the total cost of transport to meet customer needs over the planning horizon. The Inventory Routing Problem (IRP) is a supply chain planning problem based on the integration and coordination of inventory decisions and the determination of the best vehicle trips over a given planning horizon. The IRP is a generalization of the VRP which allows in addition, answer questions such as "When to deliver or collect products?" and "how much to collect or deliver for a given period of the planning horizon?". This is the place to implement a good replenishment policy and management practice such as the Vendor Managed Inventory (VMI). Among the replenishment policies [3], the Order-up-to-level (OU) and Maximum level (ML) policies are the most used. In the OU replenishment policy, all customers have a maximum storage capacity and the quantity delivered is such that the maximum level of storage is reached at each delivery. While in the ML policy all customers have a maximum storage capacity and the quantity delivered is such that the maximum level of storage is not exceeded at each delivery [4] . Regarding the VMI, it is a practice in which the supplier decides when and how much to deliver to the customer based on customers' inventory information. He must also ensure that there is no stock shortage at the customers. This practice contrasts with the Retailer Managed Inventory (RMI) or Costumer Managed Inventory (CMI) practice in which customers decide the time and amount of replenishment, regardless of decisions made by other customers and the supplier. The CMI or RMI is therefore an appropriate practice for VRP. For a comparative study between the CMI and the VMI see [4]. In a supply chain, the production problem is solved independently of the decisions that characterize the VRP or the IRP. Similarly, the VRP or IRP problem is analysed without considering production decisions. However in a comparative study to analyse the importance of coordination of production and distribution [5] , it has been shown that savings of $3 \%$ to $20 \%$ on the total cost of production and distribution can be achieved by considering the supply chain as an integrated and coordinated system. As a result, integrating and coordinating production and distribution decisions has become a major concern for researchers in recent decades. The integrated and coordinated planning of the production and distribution functions of the supply chain is known as the Production Routing Problem (PRP). In order to facilitate the understanding of this work, the following organization is adopted. In Section II, a review of the PRP literature and these methods of solving are presented. Section III provides a description of the problem and its mathematical formulation. Sections IV and V propose a Branch-and-Cut algorithm and a Two-Phases Decomposition Heuristic, respectively. Test results can be found in Section VI. The conclusion of the work is presented in Section VII.

\section{LITERATURE REVIEWS}

PRP is a NP-Hard problem considering that it contains the VRP. Then, several heuristics have been developed for its 
resolution. Without being exhaustive, there are the Greedy Randomized Adaptive Search Procedure (GRASP) [6], [7], the Adaptive Large Neighborhood Search (ALNS) procedure [8], [9], the Tabu research (TS) [10], the Variable Neighborhood Search (VNS) [11], the Memetic Algorithm [12] ,the heuristic based on Mixed Integer Programming (MIP) and iterative MIP [13], [14] .Decomposition heuristics are resolution approaches that consist in dividing the problem into several subproblems. the subproblems are then solved sequentially. An improvement procedure can be used to improve the final solution obtained. Several authors have developed a decomposition approach to solve the PRP problem. The first decomposition method for PRP problems was proposed by [5], [15] to solve a PRP problem with several products. The authors cut the problem into Capacitated LSP solved to the optimal and a distribution planning problem. In the work of [16], the PRP problem is solved in two phases. The first phase consists in solving the LSP in which distribution is considered through direct shipment. Once the decisions of LSP have been made and the customers to be visited have been determined, the second phase aims to determine the route of each vehicle over the planning horizon. The authors developed a VRP heuristic to solve the second phase of the problem. An improved version of the decomposition method has been proposed by [17]. The authors used the economic algorithm of Wagner and Whitin [18] to solve LSP and the algorithm of Clarke and Wright [19] for the construction of distribution plans for each period .They also used a local search procedure to improve the solution. Since the introduction of the Branch-and-cut algorithm (B\&C) as an exact method to solve the integrated IRP [20], more and more researchers have been interested in the exact resolution of the PRP . the B \& $\mathrm{C}$ is the most widely used of the exact methods of resolution of the PRP. It has been used to solve a PRP problem in which vehicle capacity and plant production capacity have not been considered [21]. For the model with the consideration of the capacity of a single vehicle used to transport a single type of product and in which production capacity is not considered, see [14]. The singlevehicle model has been extended to the multi-vehicle model in [8]. Qiu and al. have recently introduced three B\&C algorithms to deal with various PRP. The first is a PRP in which reverse logistics and remanufacturing are considered [22] , the second is a problem of production and distribution of perishable products [23] and the third is a problem of production of several types of products with the use of several homogeneous vehicles and taking into account the setting cost when there is production or when moving from the production of one product to another [24]. For the exact method using Bender's decomposition see [25]. The difficulty in the exact resolution of the PRP lies in eliminating subtours in a vehicle's route. This difficulty is the same for any tour problem such as TSP, VRP or IRP. Two subtour elimination constraints (SECs) are increasingly used in PRP. On the one hand, there are the SECs resulting from the formulation of Boudia and al. [6], [17] and on the other hand, the SECs developed for selective TPS in [26] . Three methods or callable libraries have been proposed for the exact or heuristic separation of these SECs. Among these methods, there are four heuristics developed at the base for solving the Capacitated VRP (CVRP) with consideration of vehicle capacity [27] . An exact and heuristic separation by using the minimum S-T cut algorithm of the Concorde callable library have been developed by [28], [29] and a polyhedral approach to SECs separation has also been proposed in [30] . The exact algorithms used for PRP resolution in most cases use one of these SECs separations approaches. Table I presents the works on the use of exact resolution methods as well as the type of mathematical formulation of the model and the separation method used. The notation F|k refers to the formulation with vehicle index and the formulation $F \mid n k$ refers to the formulation without vehicle index. These notations can be enriched by the precision of the type of procurement policy used in the mathematical formulation of the model. Thus, with the OR replenishment policy, one can obtain the notation of type $\mathrm{F}(\mathrm{OU}) \mid \mathrm{k}$ and $\mathrm{F}(\mathrm{OU}) \mid \mathrm{nk}$ and the notation of type $\mathrm{F}(\mathrm{ML}) \mid \mathrm{k}$ and $\mathrm{F}(\mathrm{ML}) \mid \mathrm{nk}$ for the ML replenishment policies. See [8] for details on the notation of mathematical formulations. Readers are also invited to see a very detailed literature review proposed in [31]. In this literature review, the authors presented the different types of integration problems such as the integrated problem of LSP and direct delivery, IRP, and the PRP.

TABLE. I. SUbTOUR Elimination PROCEDURES

\begin{tabular}{|l|l|l|l|}
\hline Works & Model & Method of resolution & Separation method used \\
\hline Adulyasak and al.[25] & F|k & Bender's decomposition & Applegate and al.[28] \\
Ruokokoski and al.[21] & F|nk & B\&C & Applegate and al.[32], [29] \\
Archetti and al.[14] & F|nk & B\&C & Padberg and Rinaldi .[30] \\
Adulyasak and al.[8] & F|k and F|nk & B\&C & Applegate and al.[28] \\
Qiu and al.[22] & F|k & B\&C & Lysgaard and al.[27] \\
Qiu and al.[23] & F|nk & B\&C & Lysgaard and al.[27] \\
Qiu and al.[24] & F|nk & B\&C & Lysgaard and al.[27] \\
\hline
\end{tabular}


They also highlighted the different mathematical formulations of the PRP with resolution methods. A classification based on four criteria and covering 77 research studies from 1993 to 2016 was presented in a literature review [33]. The authors categorized the work according to the level of decision, the typology of the problem in the supply chain, the type of objective sought and the problem optimization model (resolution method). The problem studied in this work is to plan and optimize an integrated supply chain in which production decisions for a single type of product in a plant with no storage capacity and those for inventories in an offplant depot are integrated and coordinated to meet the deterministic demands of several customers. We denote this problem by the "External Depot Production Routing Problem" (EDPRP), To the best of our knowledge, the PRP with External Depot has not been addressed before. In this EDPRP, a fleet of homogeneous vehicles leaves the depot for the distribution of products to customers or the collection of products at the plant. In the following section, more detailed description of the model and its mathematical formulation will be presented.

\section{DESCRIPTION OF THE PROBLEM AND MATHEMATICAL FORMULATION}

$\mathrm{G}=(\mathrm{N}, \mathrm{A})$ is a complete graph in which $\mathrm{N}$ represents all the nodes formed by the plant, the depot and the customers with the index $i \in\{0 \ldots n+1\}$ and $A(N)=\{(i, j): i, j \in N, i \neq j\}$ all the $\operatorname{arcs}$ in $\mathrm{N}$. The plant is represented by $\mathrm{n}+1$, the depot is indexed by 0 and all customers are represented by $\{1, \ldots, n\}$. the graph of Fig. 1 represents the collection and distribution network for one plant, one depot and seven customers.

The sets

Let denote $N_{c}=\{1, \ldots, \mathrm{n}\}$ the set of customers,

$N_{d c}=\{0, \ldots, \mathrm{n}\}$ the set consisting of the depot and customers,

$N_{c u}=\{1, \ldots, \mathrm{n}+1\}$ the set consisting of the customers and the plant,

$N=\{0, \ldots, \mathrm{n}+1\}$ the set for depot, customers and plant,

$\mathrm{T}=\{1, \ldots, 1\}$ the set of periods (days) of the planning horizon,

$\mathrm{K}=\{1, \ldots, \mathrm{m}\}$ the set of homogeneous fleet of vehicles.

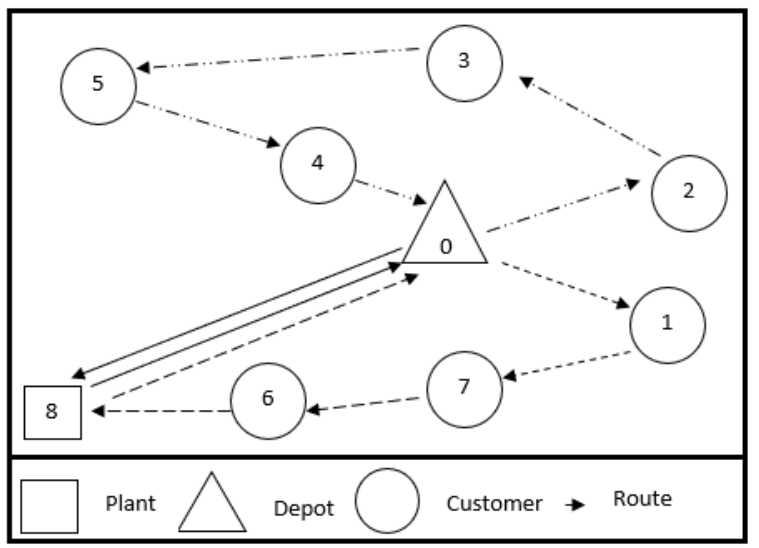

Fig. 1. Collection and Distribution Network.
The index:

$\mathrm{i}$ and $\mathrm{j}$ represent the index for the nodes of $\mathrm{N}$,

$t$ is the index of the different periods of the planning horizon, $\mathrm{k}$ is the index for the homogeneous fleet of vehicles.

The parameters:

$u$ : Unit Production Cost (UPC),

f: fixed cost of production,

$h_{i}:$ Unit Inventory Cost (UIC) at node $\mathrm{i}$,

$c_{i j}$ : Unit Transportation Cost (UTC) from the node $\mathrm{i}$ to the node $\mathrm{j}$,

$d_{i t}:$ the demand of the customer $\mathrm{i}$ in the period $\mathrm{t}$,

C: plant production capacity,

Q: Maximum vehicle capacity,

$L_{i}:$ Maximum or targeted storage capacity at node I,

$I_{i 0}:$ Initial stock available at node I,

$M U_{t} ;=\min \left\{\mathrm{C}, \sum_{i \in N_{c}} \sum_{\tau=t}^{l} d_{i \tau}\right\} \quad \forall \mathrm{t} \in \mathrm{T}$,

$M C_{i t}:=\min \left\{L_{i}, \mathrm{Q}, \sum_{\tau=t}^{l} d_{i \tau}\right\} \forall \mathrm{i} \in N_{1}, \forall \mathrm{t} \in \mathrm{T}$,

Decision variables

$p_{t}$ : quantity produced during the period $\mathrm{t}$

$I_{i t}$ : level of stocks at the node $\mathrm{i}$ during the period $\mathrm{t}$

$q_{i k t}$ : quantity delivered to the node $\mathrm{i}$ by vehicle $\mathrm{k}$ during the period $\mathrm{t}$

$y_{t}$ : binary variable equal to 1 if there is production at the plant or 0 if not

$Z_{i k t}$ : binary variable, equal to 1 if the i node is visited by vehicle $\mathrm{k}$ during period $\mathrm{t}$ or 0 if not

$x_{i j k t}$ : binary variable, equal to 1 if vehicle $\mathrm{k}$ travels directly from the node $\mathrm{i}$ to the node $\mathrm{j}$ during period $\mathrm{t}$ or 0 if not.

Mathematical formulation

$$
\begin{aligned}
& \mathrm{Z}=\min \sum_{t \in T}\left(u p_{t}+f y_{t}\right)+\sum_{t \in T} \sum_{i \in N} h_{i} I_{i t}+ \\
& \sum_{t \in T} \sum_{(i, j) \in A} \sum_{k \in K} c_{i j} x_{i j k t} \\
& \text { S.t } p_{t} \leq M U_{t} y_{t} \forall \mathrm{t} \in \mathrm{T} \\
& p_{t}=\sum_{k \in K} q_{0 k t} \forall \mathrm{t} \in \mathrm{T} \\
& \sum_{k \in K} z_{n+1, k t} \leq \mathrm{m} y_{t} \forall \mathrm{t} \in \mathrm{T} \\
& I_{0, t-1}+p_{t}=I_{0 t}+\sum_{i \in N_{c}} \sum_{k \in K} q_{i k t} \quad \forall \mathrm{t} \in \mathrm{T} \\
& I_{i t-1}+\sum_{k \in K} q_{i k t}=d_{i t}+I_{i t} \forall \mathrm{i} \in N_{c}, \forall \mathrm{t} \in \\
& \sum_{i \in N_{c}} \sum_{k \in K} q_{i k t} \leq I_{0 t-1} \forall \mathrm{t} \in \mathrm{T} \\
& I_{i t} \leq L_{i} \quad \forall \mathrm{i} \in N_{d c}, \forall \mathrm{t} \in \mathrm{T} \\
& \sum_{i \in N_{c}} q_{i k t} \leq \mathrm{Q} z_{0 k t} \forall \mathrm{k} \in \mathrm{K}, \forall \mathrm{t} \in \mathrm{T}
\end{aligned}
$$


$q_{0 k t} \leq \mathrm{Q} z_{n+1, k t} \quad \forall \mathrm{k} \in \mathrm{K}, \forall \mathrm{t} \in \mathrm{T}$

$q_{i k t} \leq M C_{i t} z_{i k t} \forall \mathrm{i} \in N_{c}, \mathrm{Vk} \in \mathrm{K}, \mathrm{Vt} \in \mathrm{T}$

$q_{n+1, k t}=0 \quad \forall \mathrm{k} \in \mathrm{K}, \forall \mathrm{t} \in \mathrm{T}$

$\sum_{j \in N} x_{i j k t}=z_{i k t} \forall \mathrm{i} \in \mathrm{N}, \forall \mathrm{k} \in \mathrm{K}, \forall \mathrm{t} \in \mathrm{T}$

$\sum_{j \in N} x_{j i k t}+\sum_{j \in N} x_{i j k t}=2 z_{i k t} \forall \mathrm{i} \in \mathrm{N}, \forall \mathrm{k} \in \mathrm{K}, \forall \mathrm{t} \in \mathrm{T}$

$\sum_{k \in K} z_{i k t} \leq 1 \forall \mathrm{i} \in N_{c}, \forall \mathrm{t} \in \mathrm{T}$

$x_{n+1 j k t}=0 \mathrm{Vj} \in N_{c}, \forall \mathrm{k} \in \mathrm{K}, \mathrm{\forall} \mathrm{t} \in \mathrm{T}$

$\sum_{i \in S} \sum_{j \in S} x_{i j k t} \leq|\mathrm{S}|-1 \forall \mathrm{S} \underline{\mathrm{C}} N_{c},|\mathrm{~S}| \geq 2, \forall \mathrm{k} \in \mathrm{K}, \forall \mathrm{t} \in \mathrm{T}$

$p_{t}, I_{i t}, q_{i k t} \geq 0 \mathrm{Vi} \in, \mathrm{Vk} \in \mathrm{K}, \mathrm{V} \mathrm{t} \in \mathrm{T}$

$y_{t}, x_{i j k t}, z_{i k t} \in\{0,1\} \forall \mathrm{i}, \mathrm{j} \in, \forall \mathrm{k} \in \mathrm{K}, \forall \mathrm{t} \in \mathrm{T}$

The function (1) is the objective function. This function minimizes the total cost of production, inventory and transportation. Constraints (2) determine whether there is production at a given time in the planning horizon while determining the maximum amount to be produced for that same period. The constraints (3) indicate that all quantities produced at the plant must be transported to the depot to be stored. The constraints (4) limit the number of vehicles assigned to collect products at the plant when production occurs. Constraints (5) and (6) are the constraints of product flow conservation respectively at the plant and among customers. The constraints (7) indicate that customer deliveries in each period must be made from the depot stock of the previous period. Constraints (8) limit the stocks of each period by a maximum storage capacity both at the depot and at customers. Constraints (9) indicate that the amount of product delivered by each vehicle may not exceed the maximum capacity of the vehicle. The constraints (10) indicate that the amount collected by a vehicle over a period cannot exceed the maximum capacity of the vehicle. The constraints (11) limits the quantities delivered to each customer by each vehicle over each period. Constraints (12) indicate that each vehicle passing through the plant must be empty before entering the plant. (13) and (14) are vehicles flow conservation constraints. The constraints (15) indicate that each customer is visited no more than once by a vehicle during a given period. Constraints (16) indicate that no visit from a customer is allowed when leaving the plant. Constraints (17) are Subtour Elimination Constraints. The constraints (18) are non-negativity constraints. Two resolution approaches are used to solve the problem. At first, a B\&C algorithm is developed to solve small instances and then a Tow Phases Decomposition Heuristic (TPDH) is developed for solving all instances of the problem.

\section{A B AND C ALGORITHM FOR EDPRP RESOLUTION}

For this first resolution of the model, a B\&C algorithm is used and following valid inequalities and steps are adopted:

\section{A. Valid Inequalities}

1) $\sum_{k \in K} \sum_{t \in T} z_{i k t} \geq 1 \quad \forall \mathrm{i} \in N_{c} \quad$ (20). these constraints indicate that each customer must be visited at least once on the planning horizon.
2) $z_{i k t} \leq z_{0 k t} \quad \forall \mathrm{i} \in N_{c u}, \forall \mathrm{k} \in \mathrm{K}, \forall \mathrm{t} \in \mathrm{T}$ (21). these constraints indicate that if a vehicle $\mathrm{k}$ does not leave depot 0 in period $t$, then it will not visit any customers or the plant in the same period.

3) $x_{i j k t} \leq z_{i k t}$ and $x_{i j k t} \leq z_{j k t} \quad \mathrm{~V}(\mathrm{i}, \mathrm{j})$ $\in \mathrm{A}(N), \forall \mathrm{k} \in \mathrm{K}, \forall \mathrm{t} \in \mathrm{T}(22)$. these constraints indicate that no path will enter or leave a customer if the customer is not visited for a given period.

4) $x_{i j k t} \leq q_{j k t}$ and $x_{n+1,0 k t} \leq q_{0 k t} \forall \mathrm{i} \in N_{d c}$, $\mathrm{V} \mathrm{j} \in N_{c}, \mathrm{~V} \mathrm{k} \in \mathrm{K}, \forall \mathrm{t} \in \mathrm{T}$ (23). These constraints make it possible to avoid unladen visits of vehicles.

5) $x_{i j k t}+x_{j i k t} \leq 1 \quad \forall(\mathrm{i}, \mathrm{j}) \in \mathrm{A}\left(N_{c}\right), \forall \mathrm{k} \in \mathrm{K}, \mathrm{V}, \mathrm{t} \in \mathrm{T}$ (24) . with these constraints, each arc is crossed only once and in one direction by a vehicle.

6) $z_{0, k+1, t} \leq z_{0 k t} \quad \forall \mathrm{k} \in 1, \ldots, \mathrm{m}-1, \forall \mathrm{t} \in \mathrm{T}$ (25) are the Symmetry-Breaking Constraints (SBC: valid for a homogeneous vehicles) [34], [35]. These inequities ensure that the $\mathrm{k}-1$ vehicle cannot leave the depot if the $\mathrm{k}$ vehicle is not used.

7) $I_{i, t-s-1} \geq \sum_{j=0}^{s} d_{i, t-j}\left(1-\sum_{k \in \mathrm{K}} \sum_{j=0}^{s} z_{i, k, t-j}\right)$ $\forall i \in N_{c}, \forall t \in T, \forall k \in K, s=0,1, \ldots, t-1$ (26) [14], [20].

\section{B. B and C Algorithm}

To solve the problem, constraints (20), (21), (22), (23), (24), (24), (25), (26) are added to the initial model defined by the objective function (1) and constraints (2) - (19) and let the SECs (17) drop .The relaxation of the linear program (LP) is then resolved. the approach to eliminate subtours is divided into two stages. At each node of the B\&C tree, a check in the first step whether the tour of a vehicle $\mathrm{k}$ at a date $\mathrm{t}$ contains a subtour is done. Then, in the second step the corresponding SECs and Relinking Constraints (RCs) for this vehicle $\mathrm{k}$ at date $t$ are added when a subtour is detected. In the remainder of this section, the use of "*" refers to a component of the LP solution at a node of the $\mathrm{B} \& \mathrm{C}$ search tree.

\section{Subtour Detection}

The subtour detection algorithm takes as input the vectors $Z^{*}$ and $x^{*}$ and produces as output the sets $S T_{k t}^{*}$ of customers involved in a subtour for vehicle $\mathrm{k}$ at period $\mathrm{t}$ (if this set exists). The method for determining $S T_{k t}^{*}$ is described as follows. For any vector $Z^{*}$ and $x^{*}$ of the LP solution at period $\mathrm{t}$ and for vehicle $\mathrm{k}$, the corresponding graph $G_{k t}^{*}\left(N_{k t}^{*}, A_{k t}\right)$ is defined with $N_{k t}^{*}=\left\{\mathrm{i} \in N: Z_{i k t}^{*}>0\right\}$ and,$A_{k t}\left(N_{k t}^{*}\right)=\{(\mathrm{i}$ ,j) $\left.\in A\left(N_{k t}^{*}\right): x_{i j k t}^{*}>0\right\}$. Let $S T_{0 k t}^{*}$ be the set of vertexes whose arcs form the Hamiltonian cycle of $G_{k t}^{*}$ passing through $\mathrm{i}=0$. Note $S T_{0 k t}^{*}=\left\{\mathrm{i} \in N_{k t}^{*}: 0 \in N_{k t}^{*}\right\}$ such a set. Building the sets $N_{k t}^{*}$ and $S T_{0 k t}^{*}$ are described by the Fig. 2 and Fig. 3.

Then, let define $S T_{k t}^{*}=\left\{\mathrm{i} \in N_{k t}^{*}: \mathrm{i} \notin S T_{0 k t}^{*}\right\}=N_{k t}^{*} / S T_{0 k t}^{*}$ with $S T_{0 k t}^{*} \cap S T_{k t}^{*}=\{\varnothing\}$ and $N_{k t}^{*}=S T_{0 k t}^{*} \cup S T_{k t}^{*}$. Two cycles have thus been defined. The main cycle $S T_{0 k t}^{*}$ and the $S T_{k t}^{*}$ subtour to be eliminated. Detecting a subtour in the route of a vehicle $\mathrm{k}$ during period $\mathrm{t}$ is therefore equivalent to building $S T_{k t}^{*}$. if $\left|S T_{k t}^{*}\right| \geq 2$ then a subtour is detected in the route of vehicle $\mathrm{k}$ during period t. To eliminate this subtour, the following procedure will be adopted. 


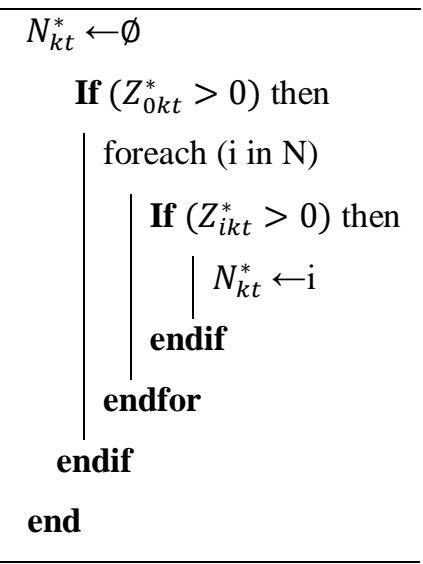

Fig. 2. The Procedure to Build $N_{k t}^{*}$.

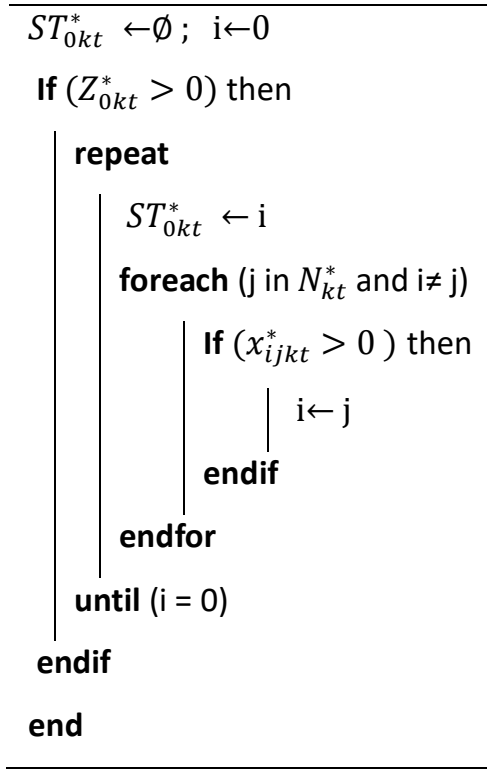

Fig. 3. The Procedure to Build Built $S T_{0 k t}^{*}$.

\section{Adding SECs}

if $\left|S T_{k t}^{*}\right| \geq 2$ then the constraints $\sum_{(i, j) \in A_{k t}\left(N_{k t}^{*}\right)} x_{i j k t} \leq$ $\left|S T_{k t}^{*}\right|-1 \quad \forall k \in K, \forall t \in T$ (27) are added.

\section{E. Adding RCs}

if $\left|S T_{k t}^{*}\right| \geq 1$ then add the constraints $\sum_{j \in S T_{0 k t}^{*}} x_{j i k t}+$ $\sum_{j \in N_{k t}^{*} /\{i\}} x_{i j k t}=2 z_{i k t} \mathrm{~V} \mathrm{k} \in \mathrm{K}, \mathrm{V} \mathrm{t} \in \mathrm{T}$, and $\mathrm{i}$ the first element of $S T_{k t}^{*}$ (28). Thanks to the constraints (28), the first element of $S T_{k t}^{*}$ is connected to an element of the main tour $\left(S T_{0 k t}^{*}\right)$ and an element of $N_{k t}^{*}$ until $S T_{k t}^{*}$ be empty. the constraints (27) and (28) are used simultaneously according to $\left|S T_{k t}^{*}\right|$ for the total elimination of subtours and isolated vertexes.

\section{F. Priority Order on Binary Variable}

Tests on six branching orders allowed us to choose the following order: connection is made on the $z_{i k t}$ variables first then to $y_{t}$ variables and finally to the $x_{i j k t}$ variables as in [24], [34].The B\&C algorithm developed here can therefore be summarized in Fig. 4 as follows:
Initialize the upper bound $\mathrm{U}^{*}$ and the incumbent solution. Initialize the node pool $\mathrm{N}$ with the root node.

Generate and insert all known valid inequalities into the program at root node of the search tree. repeat

Selection: Select the next node in N to evaluate and remove it from $\mathrm{N}$

Lower bound: Solve the LP relaxation at the current node,

let $U_{l}$ be the obtained lower bound of the current node:

if current solution is feasible then

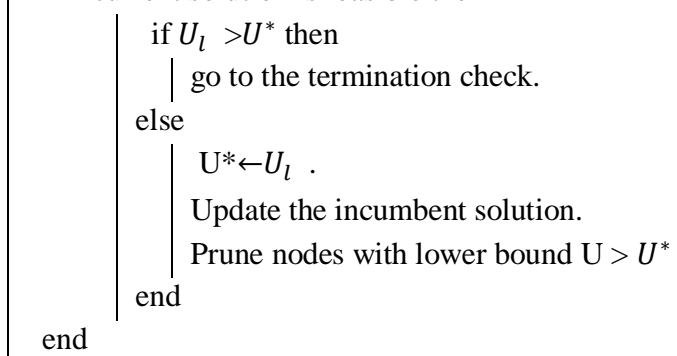

Cut generation:

foreach $\mathrm{k}$ in $\mathrm{K}$

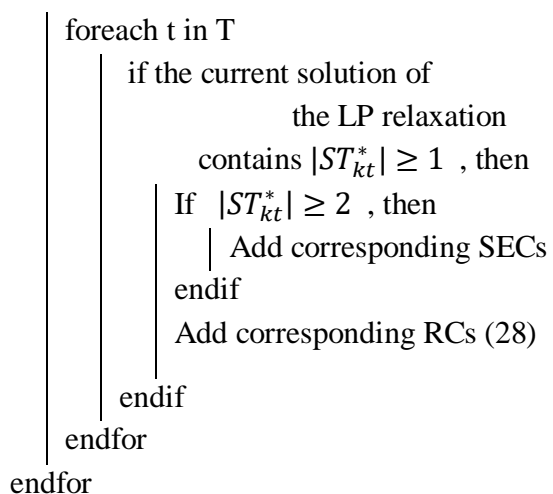

Branching: if $U_{l}>U^{*}$, go to the termination check.

until $\mathrm{N}=\varnothing$ or time limit is met (termination check) Stop with the optimal solution and the corresponding $\cos t U^{*}$.

Fig. 4. The Procedure of Branch-and-Cut.

\section{Two Phases DeCOMPosition Heuristic (TPDH) FOR EDPRP RESOLUTION}

The decomposition method used in this work to solve the EDPRP follows the same approach as in [16]. the problem is solved in two phases. In the first phase, a LP of the LSP with direct shipment and direct collection (LSP_DS\&DC) is resolved. At the end of this phase, production decisions (production and stored quantities) are set. Similarly, the quantities recovered at the plant or delivered by each vehicle to each customer over each period of the planning horizon are determined. The second phase is therefore to solve a TSP problem for each vehicle over each period of the planning horizon. So, a Genetic Algorithm (GA) is developed for the resolution of this second phase. 


\section{A. Phase I: Resolution of the LSP_DS\&DC Model}

$$
\begin{aligned}
& \mathrm{Z}=\min \\
& \sum_{t \in T}\left(u p_{t}+\right. \\
& \left.f y_{t}\right)+\sum_{t \in T} \sum_{i \in N} h_{i} I_{i t} \quad+\sum_{t \in T} \sum_{(i, j) j \in A\left({ }_{N)}\right.} \sum_{k \in K} c_{i j} x_{i j k t} \\
& \text { S.t } p_{t} \leq M U_{t} y_{t} \forall \mathrm{t} \in \mathrm{T} \\
& p_{t}=\sum_{k \in K} q_{0 k t} \forall \mathrm{t} \in \mathrm{T} \\
& \sum_{k \in K} z_{n+1, k t} \leq \mathrm{m} y_{t} \mathrm{Vt} \in \mathrm{T} \\
& I_{0, t-1}+p_{t}=I_{0 t}+\sum_{i \in N_{c}} \sum_{k \in K} q_{i k t} \mathrm{Vt} \in \mathrm{T} \\
& I_{i t-1}+\sum_{k \in K} q_{i k t}=d_{i t}+I_{i t} \mathrm{Vi} \in N_{c}, \mathrm{Vt} \in \mathrm{T} \\
& \sum_{i \in N_{c}} \sum_{k \in K} q_{i k t} \leq I_{0 t-1} \forall \mathrm{t} \in \mathrm{T} \\
& I_{i t} \leq L_{i} \forall \mathrm{i} \in N_{d c}, \mathrm{~V} \mathrm{t} \in \mathrm{T} \\
& \sum_{i \in N_{c}} q_{i k t} \leq \mathrm{Q} z_{0 k t} \forall \mathrm{k} \in \mathrm{K}, \mathrm{Vt} \in \mathrm{T} \\
& q_{0 k t} \leq \mathrm{Q} z_{n+1, k t} \forall \mathrm{k} \in \mathrm{K}, \forall \mathrm{t} \in \mathrm{T} \\
& q_{i k t} \leq M C_{i t} z_{i k t} \mathrm{Vi} \in N_{c}, \mathrm{Vk} \in \mathrm{K}, \mathrm{Vt} \in \mathrm{T} \\
& q_{n+1, k t}=0 \forall \mathrm{k} \in \mathrm{K}, \mathrm{\forall t \in T} \\
& \sum_{k \in K} z_{i k t} \leq 1 \mathrm{Vi} \in N_{c}, \mathrm{Vt} \in \mathrm{T} \\
& x_{0 i k t}+x_{i 0 k t}=2 z_{i k t} \forall \mathrm{i} \in N_{c u}, \mathrm{Vk} \in \mathrm{K}, \mathrm{Vt} \in \\
& x_{i j k t}=0 \mathrm{~V}(\mathrm{i}, \mathrm{j}) \in A\left(N_{c u}\right), \mathrm{Vk} \in \mathrm{K}, \mathrm{Vt} \in \mathrm{T} \\
& p_{t}, I_{i t}, q_{i k t} \geq 0 \mathrm{Vi} \in, \forall \mathrm{k} \in \mathrm{K}, \forall \mathrm{t} \in \mathrm{T} \\
& y_{t}, x_{i j k t}, z_{i k t} \in\{0,1\} \forall \mathrm{i}, \mathrm{j} \in, \forall \mathrm{k} \in \mathrm{K}, \mathrm{Vt} \in \mathrm{T}
\end{aligned}
$$

To the mathematical model above, constraints (20), (21), (25), (26), $z_{j k t} \leq q_{j k t}$ and $z_{n+1, k t} \leq q_{0 k t}, \forall \mathrm{j} \in N_{c}, \forall \mathrm{k} \in$ $\mathrm{K}, \mathrm{V} t \in \mathrm{T}$ (46) (to avoid unladen visits of vehicles.) are added.

The LSP_DS\&DC model is solved according to the conditions set out in the experimental section. In the second phase," **" refers to components of the LP solution from phase 1 of the decomposition method. The results concerning the visit of the factory or each customer by each vehicle over each period $\left(z_{i k t}^{* *}\right)$ are reused to serve as an entry for phase II.

\section{B. Phase II: Resolution of the TSP}

The GA for each vehicle $\mathrm{k}$ at each period $\mathrm{t}\left(G A_{k t}\right)$ is described in Fig. 5. A vector of customers is used to model each chromosome. However, the depot and the plant are only considered in the evaluation of the fitness of the chromosomes. A Roulette Wheel is used for the selection operation. An operator with one crossing point is used to carry out the crossing of the chromosomes. The $G A_{k t}$ is applied when $\left|N_{k t}^{* *} /\{0, \mathrm{n}+1\}\right|>2$. And the total cost of transportation is equal to the sum of the transportation costs of each vehicle $\mathrm{k}$ for each period $t$. The cost of a direct collection to the plant or a trip for which $1 \leq\left|N_{k t}^{* *} /\{0, \mathrm{n}+1\}\right| \leq 2$ (one or two customers visited) remains unchanged and therefore does not need an improvement by $G A_{k t}$. However, when $\left|N_{k t}^{* *} /\{0, \mathrm{n}+1\}\right|>2$, the transportation cost for the vehicle $\mathrm{k}$ in the period $\mathrm{t}$ is equal to the fitness of the best chromosome of the last generation of the population of the Procedure of $G A_{k t}$.

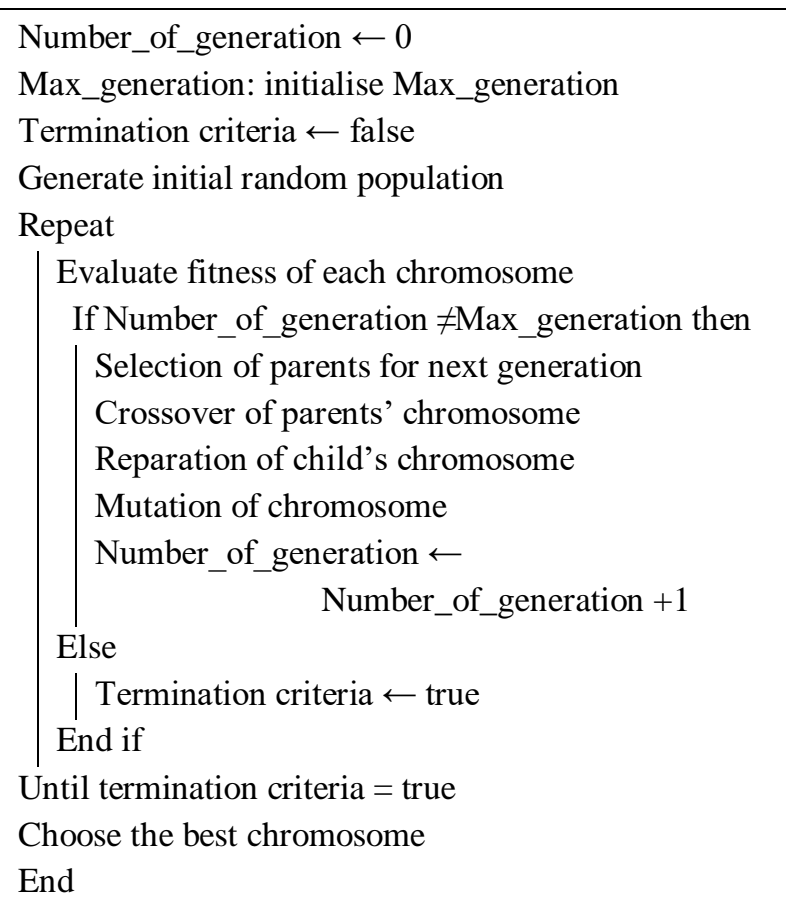

Fig. 5. The Procedure of of Genetic Algorithm on the Route of Vehicle k at Period $\mathrm{t}\left(G A_{k t}\right)$.

\section{EXPERIMENTATIONS AND RESULTS}

\section{A. Experiments}

The B\&C algorithm described in Section 4 and the decomposition heuristic in Section 5 have been implemented in C++ with CPLEX 12.6 on a 64-bit Intel Pentium Dual Core $1.60 \mathrm{GHz}, 1.60 \mathrm{GHz}$ PC with $4 \mathrm{~GB}$ RAM. Only one thread was used in the experiments and the duration of each test is limited to 7200 seconds for the $\mathrm{B} \& \mathrm{C}$ and 3600 seconds for the decomposition heuristic. The purpose of these experiments is to evaluate the effectiveness of the model. The instances used in this work are derived from an adaptation of the instances used for the Multivehicle Production and Inventory Routing Problems (MVPRP). For more details on the instances used in Table II, see [34]. Since all deliveries to customers are made basing on the quantities in stock at the previous period in depot, an initial stock at the depot greater than zero is defined, unlike the basic instances. Moreover, the depot does not have the same geographical location as the plant. Thus, the initial stock levels at the depot for all instances are set to $I_{00}=0.5 *$ $\left(\frac{\sum_{t \in T} \sum_{i \in N_{C}} d_{i t}}{l}\right)$ and the plant's position is set to $(0.0)$.

A total of 32 instances are applied to 4 classes of situations, i.e. a total of $32 \times 4=128$ instances. However, only the first $12 \times 4=48$ instances are used for the tests relating to the B\&C algorithm. The first class consists of standard instances. The second and third class have the same characteristics as the first class. However, the second class has a high unit cost of production and the third class has a high transport cost. the fourth class is like the first and second class except for the unit inventory cost which is zero. The characteristics of the classes are summarized in Table III. 
TABLE. II. CHARACTERISTICS OF THE INSTANCS FOR THE EPRP

\begin{tabular}{|l|l|l|l|l|l|}
\hline $\mathbf{n}$ & $\mathbf{l}$ & $\mathbf{m}$ & $\mathbf{C}$ & $\mathbf{L}_{\mathbf{0}}$ & $\mathbf{Q}$ \\
\hline 10 & $3 / 6$ & 2 & 304 & 152 & 198 \\
\hline 10 & $3 / 6$ & 3 & 304 & 152 & 132 \\
\hline 15 & $3 / 6$ & 2 & 470 & 235 & 198 \\
\hline 15 & $3 / 6$ & 3 & 470 & 235 & 132 \\
\hline 20 & $3 / 6$ & 2 & 540 & 270 & 283 \\
\hline 20 & $3 / 6$ & 3 & 540 & 270 & 189 \\
\hline 25 & $3 / 6$ & 2 & 700 & 350 & 283 \\
\hline 25 & $3 / 6$ & 3 & 700 & 350 & 189 \\
\hline 30 & $3 / 6$ & 3 & 768 & 384 & 228 \\
\hline 30 & $3 / 6$ & 4 & 768 & 384 & 171 \\
\hline 35 & $3 / 6$ & 3 & 948 & 474 & 276 \\
\hline 35 & $3 / 6$ & 4 & 948 & 474 & 207 \\
\hline 40 & $3 / 6$ & 3 & 1256 & 628 & 360 \\
\hline 40 & $3 / 6$ & 4 & 1256 & 628 & 216 \\
\hline 45 & 3 & 3 & 1438 & 719 & 360 \\
\hline 45 & 3 & 4 & 1438 & 719 & 207 \\
\hline 50 & 3 & 3 & 1348 & 674 & 360 \\
\hline 50 & 3 & 4 & 1348 & 674 & 270 \\
\hline & & & & & \\
\hline
\end{tabular}

TABLE. III. INSTANCE Class CHARACTERISTICS

\begin{tabular}{|l|l|l|l|}
\hline Classes & UPC & UIC & UTC \\
\hline Class 1 & Standard & Standard & Standard \\
\hline Class 2 & High & Standard & Standard \\
\hline Class 3 & Standard & Standard & High \\
\hline Class 4 & Standard & Null & Standard \\
\hline
\end{tabular}

\section{B. Results}

Let \%diff represents the percentage difference between the cost determined by the heuristic method and the B\&C method. diff is equal to the difference between the sums of the average costs obtained by the heuristic method and the B\&C method divided by the sum of the average costs of the $\mathrm{B} \& \mathrm{C}$ method. In Table IV, each line corresponds to an average of 4 instance classes results for each number of customers $n$ over 1 periods with $\mathrm{m}$ vehicles. the TOTAL Cost column represents the sum of the costs of the production cost (fixed and variable), the cost of inventories and the total cost of transport over 1 periods with $\mathrm{m}$ vehicles. The TOTAL Cost column represents the sum of the costs of the production cost (fixed and variable), the cost of inventories and the total cost of transport. the gap only available for the $\mathrm{B} \& \mathrm{C}$ refers to the percentage difference between the upper bound and the lower bound. the CPU defines the time taken to resolve the instances. 9 out of 48 instances have not been resolved and 19 out of 48 instances have not been resolved to the optimum. In the \% Diff column, 7 out of 12 average results from the results of the $\mathrm{B} \& \mathrm{C}$ are better than those of the TPDH.

TABLE. IV. AVERAGE RESULTS OF B AND C VS TPDH

\begin{tabular}{|c|c|c|c|c|c|c|c|c|c|c|}
\hline \multirow[b]{2}{*}{$n$} & \multirow[b]{2}{*}{$l$} & \multirow[b]{2}{*}{$m$} & \multicolumn{3}{|l|}{ B\&C } & \multicolumn{4}{|l|}{ TPDH } & \multirow{2}{*}{ \%diff } \\
\hline & & & TOTAL Cost & GAP & $C P U$ & TOTAL Cost & CPU MIP & $C P U A G$ & T.CPU & \\
\hline 10 & 3 & 2 & 33581.75 & 10.06 & 4.19 & 34221.25 & 0.99 & 0.54 & 1.53 & 1.90 \\
\hline 15 & 3 & 2 & $23163^{\{2,3,4\}}$ & 13.57 & 18.27 & 20028 & 2.03 & 1.89 & 3.92 & -13.53 \\
\hline 20 & 3 & 2 & $33700.5^{\{1,2\}}$ & 14.15 & 55.85 & 33463 & 1.15 & 2.18 & 3.32 & -0.70 \\
\hline 10 & 6 & 2 & 83259.5 & 0.34 & $5332.63^{2}$ & 84116.5 & 9.97 & 0.91 & 10.88 & 1.03 \\
\hline 15 & 6 & 2 & 112744.5 & 1.36 & $6698.81^{2}$ & 111004.25 & 9.93 & 1.29 & 11.22 & -1.54 \\
\hline 20 & 6 & 2 & $150748^{\{1\}}$ & 5.09 & $7201.99^{(3)}$ & 151044 & 20.8 & 1.89 & 22.69 & 0.20 \\
\hline 10 & 3 & 3 & 35600 & 3.18 & 12.9 & 35826.5 & 1.71 & 0.71 & 2.41 & 0.64 \\
\hline 15 & 3 & 3 & $48238^{\{3\}}$ & 0.59 & 126.18 & 47681 & 2.13 & 1.01 & 3.14 & -1.15 \\
\hline 20 & 3 & 3 & $85802^{\{1,3\}}$ & 0.9 & 174.75 & 70477 & 1.51 & 1.52 & 3.03 & -17.86 \\
\hline 10 & 6 & 3 & 81877.5 & 2.56 & $7202.29^{4}$ & 87065.25 & 13.03 & 0.76 & 13.79 & 6.34 \\
\hline 15 & 6 & 3 & 112395.75 & 8.9 & $7201.79^{4}$ & 115560.5 & 16.04 & 1.41 & 17.44 & 2.82 \\
\hline 20 & 6 & 3 & 128464 & 15.36 & $7201.01^{4}$ & 131567.5 & 2185.17 & 1.63 & 2186.8 & 2.42 \\
\hline \multicolumn{3}{|c|}{ Total } & \multicolumn{3}{|l|}{929574.5} & \multicolumn{4}{|l|}{922054.75} & -0.81 \\
\hline
\end{tabular}


However, the negative differences in instances 15_3_2 and instances 20_3_3 give an overall advantage to the results of the TPDH. The total difference between the TPDH result and the $\mathrm{B} \& \mathrm{C}$ is calculated as follows: TOTAL \%diff = $100 *(922054.75-929574.5) / 929574.5)$ or TOTAL \%diff = -0.81 . Based on the results of Table IV and TOTAL \%diff it can be can said that the results of the resolved instances are globally close to $99.19 \%$ (100 -| TOTAL \%diff |). Although the instances resolved with the $\mathrm{B} \& \mathrm{C}$ approach do not contain subtour, too many instances remain unresolved or unresolved to the optimum and the GAPs obtained are generally poor compared to the GAPs of the exact approaches described in Table I. However, it allows to make a comparison with the equivalent instances resolved by the TPDH. The Tables V, VI and VII describe respectively the averages results from the 128 instances with the TPDH, the percentages of production, inventory and transportation cost in the total production cost and the percentages of computation times. In these tables, Columns $\mathrm{n}, 1$ and $\mathrm{m}$ respectively refer to the number of customers, periods and vehicles, the PROD column designates the total cost of production. this total production cost consists of the fixed production cost and the variable cost of production. Then, the INV column refers to the cost of inventories, the TRANS column represents the total cost of transport. The TOTAL Cost column is the sum of the costs of production, inventories and transportation. The MIP CPU, GA CPU and TOTAL CPU columns refer respectively to the time taken for the resolution of the LSP_DS\&DC, the optimization of the transport part by the GA, and the sum of these two times. An average of 459,51 seconds (7.66 minutes) for computation time in the Table $\mathrm{V}$ is acceptable because EDPRP is a tactic levels problem. however, $99.32 \%$ of this calculation time is globally dedicated to the MIP and only $0.68 \%$ for the GA (Table VII). Thus, developing an GA or a memetic algorithm to solve the problem could considerably reduce the computation time of the different instances. The Table VI shows that a global percentage of $30.21 \%(8.59 \%+$ $21.62 \%$ ) of the total cost is allocated to storage and transport.

TABLE. V. DetaILS OF AVERAges Tests RESUlts FOR TPDH

\begin{tabular}{|c|c|c|c|c|c|c|c|c|c|}
\hline $\mathbf{n}$ & $\mathbf{L}$ & $\mathbf{m}$ & PROD & INV & TRANS & TOTAL Cost & CPU MIP & CPU AG & TOTAL CPU \\
\hline 10 & 3 & 2 & 23107.5 & 1827.75 & 9286 & 34221.25 & 0.99 & 0.54 & 1.53 \\
\hline 10 & 3 & 3 & 23107.5 & 1827.75 & 10891.25 & 35826.5 & 1.71 & 0.71 & 2.41 \\
\hline 15 & 3 & 2 & 30712.5 & 2693.25 & 13162 & 46567.75 & 2.64 & 1.73 & 4.37 \\
\hline 15 & 3 & 3 & 30712.5 & 2693.25 & 15608.75 & 49014.5 & 2.02 & 1.03 & 3.05 \\
\hline 20 & 3 & 2 & 35685 & 3321.75 & 13362.75 & 52369.5 & 1.24 & 1.98 & 3.22 \\
\hline 20 & 3 & 3 & 35685 & 3321.75 & 15991.5 & 54998.25 & 1.38 & 1.44 & 2.82 \\
\hline 25 & 3 & 2 & 39292.5 & 4389.75 & 15547.25 & 59229.5 & 1.96 & 2.79 & 4.75 \\
\hline 25 & 3 & 3 & 39292.5 & 4389.75 & 16880.5 & 60562.75 & 1.69 & 2.60 & 4.28 \\
\hline 30 & 3 & 3 & 45240 & 4209 & 19411 & 68860 & 5.93 & 3.71 & 9.64 \\
\hline 30 & 3 & 4 & 45240 & 4209 & 22833 & 72282 & 10.85 & 3.35 & 14.19 \\
\hline 35 & 3 & 3 & 66592.5 & 4662.75 & 21205 & 92460.25 & 4.10 & 3.64 & 7.74 \\
\hline 35 & 3 & 4 & 66592.5 & 4604.25 & 26470.75 & 97667.5 & 4.68 & 3.68 & 8.35 \\
\hline 40 & 3 & 3 & 69030 & 7533.5 & 22267 & 98830.5 & 3.66 & 5.34 & 9.00 \\
\hline 40 & 3 & 4 & 69030 & 7533.5 & 26193.75 & 102757.25 & 4.34 & 4.69 & 9.02 \\
\hline 45 & 3 & 3 & 91942.5 & 7550 & 24608.25 & 124100.75 & 5.21 & 6.36 & 11.57 \\
\hline 45 & 3 & 4 & 91942.5 & 7874.75 & 31284.5 & 131101.75 & 7.07 & 5.05 & 12.12 \\
\hline 50 & 3 & 3 & 73027.5 & 7767.75 & 29310.75 & 110106 & 8.56 & 8.91 & 17.47 \\
\hline 50 & 3 & 4 & 73027.5 & 7767.75 & 33060 & 113855.25 & 9.69 & 5.82 & 15.52 \\
\hline 10 & 6 & 2 & 63082.5 & 7430 & 13604 & 84116.5 & 9.97 & 0.91 & 10.88 \\
\hline 10 & 6 & 3 & 63082.5 & 7451 & 16531.75 & 87065.25 & 13.03 & 0.76 & 13.79 \\
\hline 15 & 6 & 2 & 82192.5 & 10974.75 & 17837 & 111004.25 & 9.93 & 1.29 & 11.22 \\
\hline 15 & 6 & 3 & 82192.5 & 11055.75 & 22312.25 & 115560.5 & 16.04 & 1.41 & 17.44 \\
\hline 20 & 6 & 2 & 93502.5 & 12653.25 & 20722.5 & 126878.25 & 18.20 & 2.01 & 20.21 \\
\hline 20 & 6 & 3 & 94252.5 & 12502 & 24813 & 131567.5 & 2185.17 & 1.63 & 2186.80 \\
\hline 25 & 6 & 2 & 104422.5 & 16560.25 & 21631 & 142613.75 & 19.72 & 3.23 & 22.94 \\
\hline 25 & 6 & 3 & 104422.5 & 16281.25 & 26986.75 & 147690.5 & 41.22 & 3.01 & 44.23 \\
\hline 30 & 6 & 3 & 118267.5 & 16857.75 & 33729.75 & 168855 & 140.01 & 3.03 & 143.04 \\
\hline 30 & 6 & 4 & 118267.5 & 16728 & 40578 & 175573.5 & 1327.60 & 2.52 & 1330.12 \\
\hline 35 & 6 & 3 & 145567.5 & 21020.5 & 36776 & 203364 & 2835.51 & 4.30 & 2839.81 \\
\hline 35 & 6 & 4 & 145567.5 & 20479.5 & 45154.75 & 211201.75 & 3606.21 & 3.74 & 3609.94 \\
\hline 40 & 6 & 3 & 177937.5 & 29374.25 & 39935 & 247246.75 & 1556.74 & 4.89 & 1561.64 \\
\hline 40 & 6 & 4 & 177937.5 & 28849.25 & 44744.75 & 251531.5 & 2747.24 & 4.12 & 2751.36 \\
\hline \multicolumn{3}{|c|}{ Total } & 78748,5938 & 9887,33594 & 24147,82813 & 112783,7578 & 456,38 & 3,13 & 459,51 \\
\hline
\end{tabular}


TABLE. VI. PERCENTAGe OF Production, IVENTORY AND TANSPOTATION COST FOR TPDH

\begin{tabular}{|l|l|l|l|l|l|l|}
\hline $\mathbf{n}$ & PROD & \%PROD & INV & \% INV & TRANS & \% TRANS \\
\hline 10 & 689520 & $71.46 \%$ & 74146 & $7.68 \%$ & 201252 & $20.86 \%$ \\
\hline 15 & 903240 & $70.10 \%$ & 109668 & $8.51 \%$ & 275680 & $21.39 \%$ \\
\hline 20 & 1036500 & $70.84 \%$ & 127195 & $8.69 \%$ & 299559 & $20.47 \%$ \\
\hline 30 & 1308060 & $67.35 \%$ & 168015 & $8.65 \%$ & 466207 & $24.00 \%$ \\
\hline 35 & 1697280 & $70.17 \%$ & 203068 & $8.40 \%$ & 518426 & $21.43 \%$ \\
\hline 40 & 1975740 & $70.53 \%$ & 293162 & $10.46 \%$ & 532562 & 223571 \\
\hline 45 & 735540 & $72.05 \%$ & 61699 & $6.04 \%$ & $219.01 \%$ & 249483 \\
\hline 50 & 584220 & $65.21 \%$ & 62142 & $6.94 \%$ & $27.85 \%$ & $21.62 \%$ \\
\hline Total & 8930100 & $69.79 \%$ & 1099095 & $8.59 \%$ & & 2766740 \\
\hline
\end{tabular}

TABLE. VII. PERCENTAGE OF CPU FOR TPDH

\begin{tabular}{|l|l|l|l|l|}
\hline $\mathbf{n}$ & CPU MIP & \% CPU MIP & CPU AG & \% CPU AG \\
\hline 10 & 102.78 & $89.81 \%$ & 11.661 & $10.19 \%$ \\
\hline 15 & 122.497 & $84.86 \%$ & 21.852 & $15.14 \%$ \\
\hline 20 & 8823.906 & $99.68 \%$ & 28.266 & $0.32 \%$ \\
\hline 25 & 258.308 & $84.74 \%$ & 46.499 & $15.26 \%$ \\
\hline 30 & 5937.537 & $99.16 \%$ & 50.422 & $0.84 \%$ \\
\hline 35 & 25801.949 & $99.76 \%$ & 61.458 & $0.24 \%$ \\
\hline 40 & 17247.927 & $99.56 \%$ & 76.156 & $0.44 \%$ \\
\hline 45 & 49.103 & $51.84 \%$ & 45.626 & $48.16 \%$ \\
\hline 50 & 73.009 & $55.33 \%$ & 58.942 & $44.67 \%$ \\
\hline Total & 58417.016 & $99.32 \%$ & 400,882 & $0.68 \%$ \\
\hline
\end{tabular}

\section{CONCLUSION}

This work focuses primarily on modeling a production routing problem in which the plant's storage capacity (depot) is geographically dissociated from the plant's location. The results of 48 tests with a B \& $\mathrm{C}$ approach were compared with those of a decomposition heuristic method. The average results of all 128 instances are also presented in Table V. In this study, a depot-based distribution policy is adopted. However, the lack of storage capacity at the plant does not exclude the possibility to supply customers from the plant during production days. In future work, we will improve the $\mathrm{B} \& \mathrm{C}$ algorithm used in this paper by adding an initial solution generation heuristic to overcome the infeasibility problem and if possible, an improvement phase to reinforce the results. A GA or memetic algorithm will also be developed as an overall means of solving the problem instead of using a decomposition method.

\section{REFERENCES}

[1] Y. Pochet and L. A. Wolsey, Production planning by mixed integer programming. New York; Berlin: Springer, 2006.

[2] J. K. Lenstra and A. H. G. Kan, "Complexity of vehicle routing and scheduling problems," Networks, vol. 11, no. 2, pp. 221-227, 1981.

[3] L. Bertazzi and M. G. Speranza, "Inventory routing problems: an introduction," EURO Journal on Transportation and Logistics, vol. 1, no. 4, pp. 307-326, Dec. 2012, doi: 10.1007/s13676-012-0016-7.

[4] C. Archetti and M. G. Speranza, "The inventory routing problem: the value of integration: The inventory routing problem: the value of integration," International Transactions in Operational Research, vol. 23, no. 3, pp. 393-407, May 2016, doi: 10.1111/itor.12226.

[5] P. Chandra and M. L. Fisher, "Coordination of production and distribution planning," European Journal of Operational Research, vol. 72, no. 3, pp. 503-517, Feb. 1994, doi: 10.1016/0377-2217(94)90419-7.
[6] M. Boudia, M. A. O. Louly, and C. Prins, "A reactive GRASP and path relinking for a combined production-distribution problem," Computers \& Operations Research, vol. 34, no. 11, pp. 3402-3419, Nov. 2007, doi: 10.1016/j.cor.2006.02.005.

[7] M.-S. Casas-Ramírez, J.-F. Camacho-Vallejo, R. G. González-Ramírez, J.-A. Marmolejo-Saucedo, and J.-M. Velarde-Cantú, "Optimizing a Biobjective Production-Distribution Planning Problem Using a GRASP," Complexity, 2018. [Online]. Available: https://www.hindawi.com/journals/complexity/2018/3418580/abs/. [Accessed: 04-May-2018].

[8] Y. Adulyasak, J.-F. Cordeau, and R. Jans, "Formulations and Branchand-Cut Algorithms for Multivehicle Production and Inventory Routing Problems," INFORMS Journal on Computing, vol. 26, no. 1, pp. 103120, Feb. 2014, doi: 10.1287/ijoc.2013.0550.

[9] Y. Adulyasak, J.-F. Cordeau, and R. Jans, "Optimization-Based Adaptive Large Neighborhood Search for the Production Routing Problem," Transportation Science, vol. 48, no. 1, pp. 20-45, Feb. 2014, doi: $10.1287 /$ trsc. 1120.0443 .

[10] V. A. Armentano, A. L. Shiguemoto, and A. Løkketangen, "Tabu search with path relinking for an integrated production-distribution problem," Computers \& Operations Research, vol. 38, no. 8, pp. 1199-1209, Aug. 2011, doi: 10.1016/j.cor.2010.10.026.

[11] Y. Qiu, L. Wang, X. Xu, X. Fang, and P. M. Pardalos, "A variable neighborhood search heuristic algorithm for production routing problems," Applied Soft Computing, vol. 66, pp. 311-318, May 2018, doi: 10.1016/j.asoc.2018.02.032.

[12] M. Boudia and C. Prins, "A memetic algorithm with dynamic population management for an integrated production-distribution problem," European Journal of Operational Research, vol. 195, no. 3, pp. 703-715, Jun. 2009, doi: 10.1016/j.ejor.2007.07.034.

[13] N. Absi, C. Archetti, S. Dauzère-Pérès, and D. Feillet, "A Two-Phase Iterative Heuristic Approach for the Production Routing Problem," Transportation Science, vol. 49, no. 4, pp. 784-795, Nov. 2015, doi: 10.1287/trsc.2014.0523.

[14] C. Archetti, L. Bertazzi, G. Paletta, and M. G. Speranza, "Analysis of the maximum level policy in a production-distribution system," Computers \& Operations Research, vol. 38, no. 12, pp. 1731-1746, 2011.

[15] P. Chandra, "A dynamic distribution model with warehouse and customer replenishment requirements," Journal of the Operational Research Society, vol. 44, no. 7, pp. 681-692, 1993.

[16] L. Lei, S. Liu, A. Ruszczynski, and S. Park, "On the integrated production, inventory, and distribution routing problem," IIE Transactions, vol. 38, no. 11, pp. 955-970, 2006.

[17] M. Boudia, M. A. O. Louly, and C. Prins, "Fast heuristics for a combined production planning and vehicle routing problem," Production Planning \& Control, vol. 19, no. 2, pp. 85-96, Mar. 2008, doi: $10.1080 / 09537280801893356$.

[18] H. M. Wagner and T. M. Whitin, "Dynamic Version of the Economic Lot Size Model," Management Science, vol. 5, no. 1, pp. 89-96, Oct. 1958, doi: 10.1287/mnsc.5.1.89.

[19] G. Clarke and J. W. Wright, "Scheduling of Vehicles from a Central Depot to a Number of Delivery Points," Operations Research, vol. 12, no. 4, pp. 568-581, Aug. 1964, doi: 10.1287/opre.12.4.568. 
[20] C. Archetti, L. Bertazzi, G. Laporte, and M. G. Speranza, "A branchand-cut algorithm for a vendor-managed inventory-routing problem," Transportation Science, vol. 41, no. 3, pp. 382-391, 2007.

[21] M. Ruokokoski, O. Solyali, J.-F. Cordeau, R. Jans, and H. Süral, "Efficient formulations and a branch-and-cut algorithm for a productionrouting problem," GERAD Technical Report G-2010-66, 2010.

[22] Y. Qiu, M. Ni, L. Wang, Q. Li, X. Fang, and P. M. Pardalos, "Production routing problems with reverse logistics and remanufacturing," Transportation Research Part E: Logistics and Transportation Review, vol. 111, pp. 87-100, Mar. 2018, doi: 10.1016/j.tre.2018.01.009.

[23] Y. Qiu, J. Qiao, and P. M. Pardalos, "Optimal production, replenishment, delivery, routing and inventory management policies for products with perishable inventory," Omega, Jan. 2018, doi: 10.1016/j.omega.2018.01.006.

[24] Y. Qiu, L. Wang, X. Xu, X. Fang, and P. M. Pardalos, "Formulations and branch-and-cut algorithms for multi-product multi-vehicle production routing problems with startup cost," Expert Systems with Applications, vol. 98, pp. 1-10, May 2018, doi: 10.1016/j.eswa.2018.01.006.

[25] Y. Adulyasak, J.-F. Cordeau, and R. Jans, "Benders Decomposition for Production Routing Under Demand Uncertainty,” Operations Research, vol. 63, no. 4, pp. 851-867, Aug. 2015, doi: 10.1287/opre.2015.1401.

[26] M. Gendreau, G. Laporte, and F. Semet, "A branch-and-cut algorithm for the undirected selective traveling salesman problem," Networks, vol. 32, no. 4, pp. 263-273, Dec. 1998, doi: 10.1002/(SICI)10970037(199812)32:4<263::AID-NET3>3.0.CO;2-Q.

[27] J. Lysgaard, A. N. Letchford, and R. W. Eglese, "A new branch-and-cut algorithm for the capacitated vehicle routing problem," Mathematical Programming, vol. 100, no. 2, pp. 423-445, 2004.
[28] D. Applegate, R. E. Bixby, V. Chvátal, and W. J. Cook, "The Concorde TSP Solver website. http://www.math.uwaterloo.ca/tsp/concorde.html.," 2011.

[29] D. Applegate, R. E. Bixby, V. Chvátal, and W. J. Cook, “Concorde: A code for solving traveling salesman problems. http://www.tsp.gatech.edu/concorde.html.," 2005.

[30] M. Padberg and G. Rinaldi, "A Branch-and-Cut Algorithm for the Resolution of Large-Scale Symmetric Traveling Salesman Problems," SIAM Review, vol. 33, no. 1, pp. 60-100, Mar. 1991, doi: $10.1137 / 1033004$.

[31] Y. Adulyasak, J.-F. Cordeau, and R. Jans, "The production routing problem: A review of formulations and solution algorithms," Computers \& Operations Research, vol. 55, pp. 141-152, Mar. 2015, doi: 10.1016/j.cor.2014.01.011.

[32] D. L. Applegate, R. E. Bixby, V. Chvatal, and W. J. Cook, The traveling salesman problem: a computational study The Traveling Salesman Problem: A Computational Study. Princeton University Press, Princeton, NJ. Princeton university press, 2007.

[33] Y. Kocaoğlu, A. Taşkın Gümüş, and B. Kocaoğlu, "Supply chain optimization studies: A literature review and classification," 2018.

[34] Y. Adulyasak, J.-F. Cordeau, and R. Jans, "Formulations and Branchand-Cut Algorithms for Multivehicle Production and Inventory Routing Problems," INFORMS Journal on Computing, vol. 26, no. 1, pp. 103120, Feb. 2014, doi: 10.1287/ijoc.2013.0550.

[35] L. C. Coelho and G. Laporte, "A branch-and-cut algorithm for the multiproduct multi-vehicle inventory-routing problem," International Journal of Production Research, vol. 51, no. 23-24, pp. 7156-7169, Nov. 2013, doi: 10.1080/00207543.2012.757668. 